Service social

La société québécoise en tendance, 1960-1990, par Simon Langlois, Jean-Paul Baillargeon, Gary Caldwell, Guy Fréchet, Madeleine Gauthier, Jean-Pierre Simard, Québec, IQRC, 1990.

\title{
Gérald Grandmont
}

Volume 40, numéro 2, 1991

Formation et évolution de la pratique en travail social

URI : https://id.erudit.org/iderudit/706534ar

DOI : https://doi.org/10.7202/706534ar

Aller au sommaire du numéro

Éditeur(s)

École de service social de l'Université Laval

ISSN

1708-1734 (numérique)

Découvrir la revue

Citer ce compte rendu

Grandmont, G. (1991). Compte rendu de [La société québécoise en tendance, 1960-1990, par Simon Langlois, Jean-Paul Baillargeon, Gary Caldwell, Guy Fréchet, Madeleine Gauthier, Jean-Pierre Simard, Québec, IQRC, 1990.] Service social, 40(2), 148-149. https://doi.org/10.7202/706534ar d'utilisation que vous pouvez consulter en ligne.

https://apropos.erudit.org/fr/usagers/politique-dutilisation/ 
La société québécoise en tendances 1960-1990, par Simon Langlois, Jean-Paul Baillargeon, Gary Caldwell, Guy FRÉCHET, Madeleine GAUTHIER, Jean-Pierre SIMARD, Québec, IQRC, 1990.

Cet ouvrage collectif réalisé sous la direction de Simon Langlois est un véritable ouvrage de référence. Peu le liront en entier, mais beaucoup le consulteront à plus d'une reprise. Pour ceux que préoccupe le changement social au Québec, c'est désormais un incontournable.

Langlois et son équipe y analysent 78 dimensions réunies en 17 regroupements. La tendance y est définie comme un vecteur de changement, comme un diagnostic sur le sens de l'évolution. Par une approche empirique des changements, les auteurs tentent d'établir des interrelations entre les tendances. Absence de visées normatives dans cet ouvrage; distance également avec ce que nous appelons les tendances lourdes porteuses d'avenir; différence encore d'avec un annuaire, avec ses séries statistiques. La méthode utilisée est rigoureuse; fondée qu'elle est sur l'observation de faits sur de longues périodes, les diagnostics proposés, tout en nuances et en précision à la fois, sont assurés. Leur lecture vient confirmer de nombreux autres travaux de sociologues, de même qu'elle permet, le cas échéant, d'atténuer certains de ces jugements antérieurs.

Du micro-social au marché du travail, de la stratification sociale aux modes de vie, des idéologies et croyances aux représentations sociales, des loisirs à l'intégration et à la marginalisation, cet ouvrage de plus de 600 pages fait plus que brosser un portrait des changements devenus visibles; il permet, pour peu que le lecteur se donne la peine d'aller lire les renvois, une construction du changement social qui apparaît souvent fluide, mouvant, mu par de complexes relations. II n'est pas possible de simplifier les analyses, mais il faut lire par croisements davantage que d'une manière linéaire.

Après une introduction qui présente l'approche du changement social, l'ouvrage décrit un contexte général depuis 1960 en prenant appui sur les tendances démographiques, les tendances macro-économiques et les tendances macro-technologiques. Ce chapitre est conçu pour mieux situer l'ensemble des tendances qui seront étudiées. La majorité des chapitres se terminent par les tableaux statistiques qui viennent illustrer l'examen des tendances. Les références sont également présentées à la fin de chaque chapitre. Chaque dimension traitée à l'intérieur d'un chapitre se termine par des renvois à d'autres dimensions, ce qui nous faisait dire qu'il vaut mieux consulter cet ouvrage par croisements. En annexe, on trouve une série de tableaux qui renvoient aux données de base utiles (ratios de population, taux d'emploi, indices des prix).

Cet ouvrage n'est pas un travail isolé. II s'inscrit, comme le montre Theodore Caplow dans sa préface, dans une démarche internationale qui associe actuellement la France, l'Allemagne, fédérale à l'époque, et les États-Unis. Ces pays ont publié ou le feront sous peu des ouvrages semblables. L'entreprise internationale se poursuivra par des volumes sur la Grèce, I'Espagne et l'Union soviétique. Ces pays sont réunis dans un Groupe international d'analyse du changement social dans les sociétés industrialisées, mieux connu sous le nom de Club de Québec. Le secrétariat de ce club se trouve à l'Institut québécois de recherche sur la culture. Le groupe s'est donné cinq objectifs : préparer une description d'ensemble des tendances sociales dans les sociétés industrialisées, définir les similitudes et les différences, les soumettre à une analyse com- 
parative, développer un modèle non traditionnel d'étude du changement social et établir des points de repère pour l'identification et le repérage des tendances futures.

Ce projet est ambitieux. La première étape, soit la construction de profils nationaux, est en cours. Nous aurions certes souhaité trouver dès à présent certaines comparaisons internationales, mais la chose est prématurée, cela se conçoit. Ces travaux, patients et méthodiques, sauront-ils se continuer, trouver les ressources pour atteindre les objectifs fixés par le Club de Québec ? On se prend à le souhaiter.

Comme on se prend à souhaiter un lieu de synthèse dans les gouvernements qui favorise une vision large et éclairée plutôt qu'un pilotage à vue ou qu'une " myopie " comme le signale Jean-Michel Bélorgey dans le numéro de Futuribles de mai 1991. Un lieu de synthèse qui puise davantage à de semblables travaux qu'à de vagues ou immédiates analyses de consultants.

\section{Gérald Grandmont}

Directeur de la recherche et de l'évaluation Musée de la civilisation, Québec

\section{Familles-providence - La part de l'État, par Frédéric LESEMANN et Claire CHAUME, Montréal, Éditions Saint-Martin, 1989, 286 pages.}

Vivant désormais à l'enseigne de l'État responsable, et dans une perspective globale de bien-être préconisant davantage le maintien à domicile des personnes âgées, il apparaît prudent et nécessaire de faire le point en ce qui concerne les « moyens " du " gérontin » et la perception réaliste que doit avoir l'indispensable personne-soutien.

Car les auteurs établissent bien que : " nous sommes partis d'un Étatprovidence en crise qui fait appel à la part des familles..." pour rencontrer plutôt : "des familles-providence qui comptent sur la part de l'État ".

La présente recherche, effectuée en milieu urbain, touche à la situation de quelque 200 familles prenant soin à domicile de l'un de ses membres âgés.

On fournit ainsi à des "personnes-soutiens " l'occasion privilégiée de $s^{\prime}$ exprimer à propos de questions qui les concernent de près, et cela dans le but précis de faire " connaître et reconnaître " la réalité quotidienne et les besoins particuliers des aidants.

En somme, quelles sont les occupations et les préoccupations journalières d'une personne qui prend soin d'un proche à la maison ?

Quelles sont les conséquences d'une telle responsabilité sur la santé, l'emploi du temps, les éventuelles "difficultés " de cohabitation et d'engagement? facile?

Existe-t-il des moyens qui peuvent ou pourraient rendre la tâche plus

Comment perçoit-on ce que fait ou pourrait accomplir le gouvernement afin d'aider si possible plus efficacement les personnes-soutiens et les person. nes à domicile ? 